\title{
Extreme Rituals as Social Technologies
}

\author{
Ronald Fischer
}

Centre for Applied Cross-Cultural Research, Victoria University Wellington, Wellington New Zealand

Corresponding author, e-mail:Ronald.Fischer@vuw.ac.nz

\author{
Dimitris Xygalatas \\ Interactive Minds Centre, Department of Culture and Society, \\ Aarhus University, Aarhus, Denmark \\ Department of Anthropology, University of Connecticut, Storrs, CT, USA
}

\begin{abstract}
We often think of pain as intrinsically bad, and the avoidance of pain is a fundamental evolutionary drive of all species. How can we then explain widespread cultural practices like certain rituals that involve the voluntary infliction of physical pain? In this paper, we argue that inflicting and experiencing pain in a ritual setting may serve important psychological and social functions. By providing psychological relief and leading to stronger identification with the group, such practices may result in a positive feedback loop, which serves both to increase the social cohesion of the community and the continuation of the ritual practices themselves. We argue that although the selective advantage of participation lies at the individual level, the benefits of those practices de facto extend to the group level, thereby allowing extreme rituals to function as effective social technologies.
\end{abstract}

\section{Keywords}

Ritual - pain - suffering - experimental anthropology - evolution 


\section{Introduction}

Humans are a rare biological species by having both the most intense sense of individuality and also some of the highest forms of social cohesion that are encountered in nature. No other species has the same ability to combine individual and independent agency with self-denying altruism and prosocial behaviour for the benefit of the collective. What are some of the potential mechanisms that help bond individual persons into strong cohesive groups? Whatever these mechanisms may be, they must be deeply rooted in our evolutionary past and must involve highly effective social technologies derived from our common biology.

A second anomaly of the human species is the tendency to inflict harm on oneself. Other animals may inflict harm on others, but to the best of our knowledge, only humans voluntarily and relatively frequently inflict grave bodily harm and injury to themselves. Avoiding suffering (e.g., physical and emotional pain or discomfort) is a fundamental evolutionary drive of all biological entities. Any organism will be motivated to avoid negative bodily states and will attempt to maximize pleasant states to the extent that is not interfering with other biological necessities (e.g., mating, sourcing of food). Here we propose that these two evolutionary puzzles of social bonding and self-inflicted suffering may actually be related. We argue that inflicting and experiencing negative states in social settings may serve as a particular social technology that humans have co-opted for creating cohesive in-groups.

Our argument goes beyond standard treatments of religion that have been labelled variously as charismatic signalling, costly signalling, or costly displays, by specifically focusing on the role of pain. We argue that pain and suffering are specific, biologically rooted emotional experiences that are particularly powerful in binding groups together. Extending previous arguments and mathematical models of costly signalling, we present and review empirical data from multiple field studies that provide some support for our theorizing.

\section{Pain, Fatigue and Suffering as Psycho-Biological Processes}

There is emerging consensus that the psychological experience of pain and fatigue as well as other emotional reactions associated with human suffering are not directly correlated with objective physical and physiological triggers (St Clair Gibson et al., 2003; Melzack, 2005). Gate-control theory (Melzack and Wall, 1965) and the Neuromatrix theory of pain (Melzack, 2005) have 
profoundly changed our understanding of pain. Nerve impulses arriving from the periphery are processed across various brain areas in the thalamus, limbic system and cortex. Sensory input is processed in parallel across these brain networks and sensory, affective and cognitive information from these various areas is integrated into the subjective perception of pain and suffering. As a result, pain as a psychological experience is not directly related to the physical damage to tissue or bone that may occur during extreme collective rituals. Similarly, a reticular-activiating hypofrontality model of acute exercise (Dietrich and Audiffren, 2011) proposes that endurance activities lead to a disengagement of higher-order prefrontal cortex areas and down-regulate emotional processing, but do not compromise the functioning of implicit systems related to optimal motor execution. This can help explain why individuals engaged in strenuous and high-intensity activities (and often in combination with restrictions in food and fluid intake) will continue to operate at a physical level (e.g., walking long-distances, pulling or carrying heavy objects), but are unable to process more complex information or experience emotional reactions of pain or discomfort that would be experienced in other circumstances. Therefore, the experience of body sensations related to pain, suffering or exhaustion is regulated through complex psycho-biological processes.

By extention, pain is likely linked to psychological motivations. One classic observation has been the ritualistic cleansing and purification of the body through pain. For example, Bastian et al. (2011) demonstrated that individuals who recalled an episode in which they had committed some unethical behavior were more likely to induce pain to themselves compared to people who did not recall such memories. Furthermore, the pain was rated as more intense in the moral digression condition compared to the control condition. Importantly, this experience of pain reduced guilt compared to a condition where people were not given the option of hurting themselves. This suggests that (a) individuals who believe that they have transgressed some moral boundaries are more likely to induce pain on themselves, which, in turn, (b) is experienced as more painful and, therefore, (c) allows some cleansing for the transgressions: by hurting myself, I am purifying my body and become an acceptable member of my moral community again.

These findings lead to two interim conclusions: (1) pain is a highly subjective experience that is not directly correlated with the physical or physiological condition of the body and (2) individuals may seek pain to achieve personal or social purposes. 


\section{Pain in Extreme Rituals: Moving Research to the Field}

The previous section focused on the psychological individual-level processes involved in pain and suffering. However, many collective rituals involve seemingly painful, unpleasant and dysphoric practices. This widespread practice of pain has intrigued scientists and philosophers for decades. One longstanding speculation has been that extreme rituals serve as a social bonding mechanism linking individuals to collectives. A number of theoretical models have been developed that suggest that costly behaviour serves as a signalling devise that binds communities together. Yet, no empirical proof of such mechanisms has been provided. In a field experiment, Xygalatas et al. (2013) studied naturally occurring rituals that varied in terms of levels of intensity for participants. A low-ordeal ritual a few days before the main ritual involved praying and chanting for several hours inside a Hindu temple and circling the temple in unison. The main event was a high ordeal ritual in which participants were pierced with needles (Figure 1) and skewers at a riverbank on the outskirts of the town and then carried large bamboo shrines called "kavadi" or dragged chariots attached to their skin to the same mountain temple. This

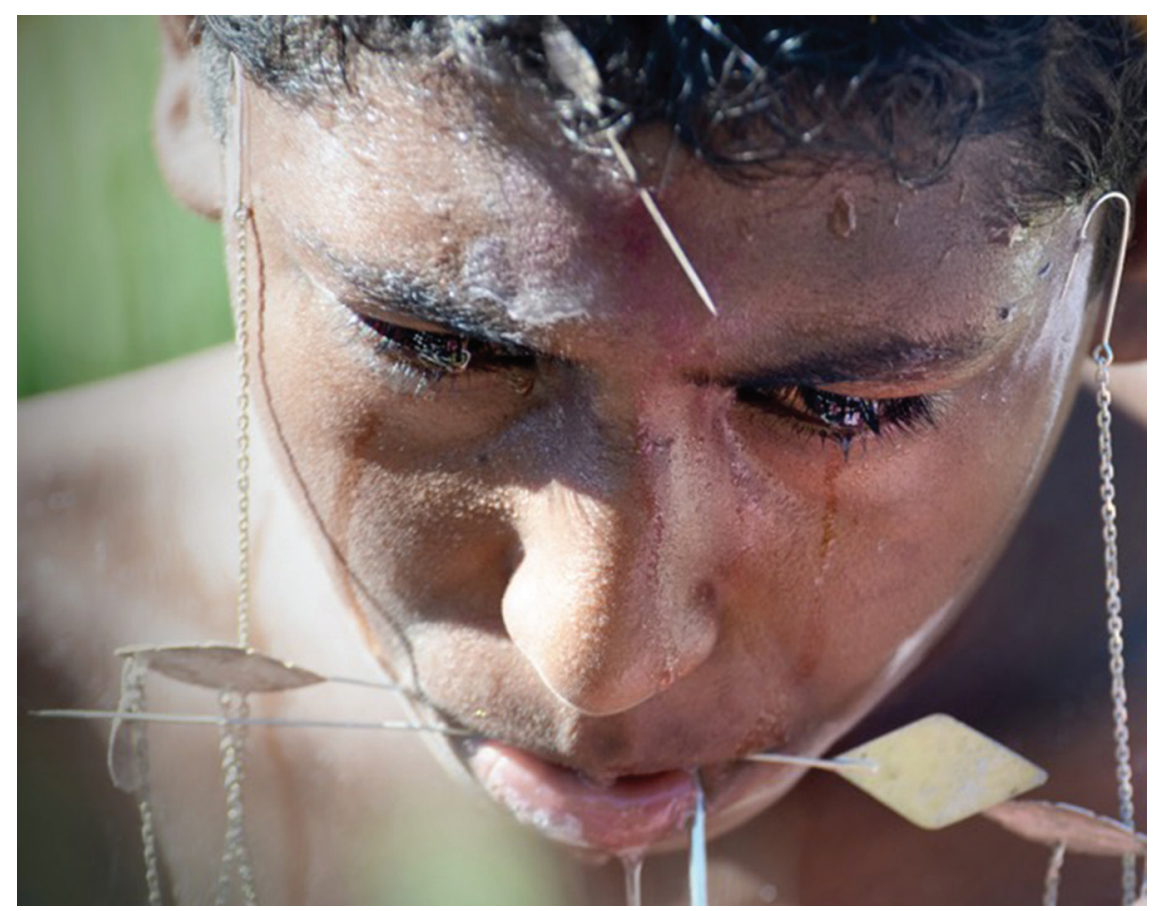

FIGURE 1 Painful rituals: a Hindu devotee in tears as he is being pierced for the Kavadi. 
procession occurs during the hottest part of the year, under the scortching sun, with the participants walking barefoot over hot asphalt and climbing the mountain without eating or drinking. Xygalatas et al. (2013) compared those who participated in the low ordeal ritual with both participants and observers in the high-ordeal ritual in a quasi-experimental design. Although random assignment was obviously not possible in a naturalistic study, the setting or the ritual allowed for random selection, i.e., each subject took part in both rituals but participated in the study only in one instance.

Individuals answered a number of short questions and then were given a substantial amount of money, equal to app. two days' wages. Before leaving the temple grounds, they were offered a chance to donate any amount of that money to the temple. The researchers tracked how much money was donated through individually marked envelopes that could be linked back to their previous answers while maintaining complete anonymity. An analysis of variance (ANOVA) revealed a main effect of group on donation $F(2,83)=11.78$, $p<0.001$ (Figure 2). Specifically, individuals (both observers and participants)

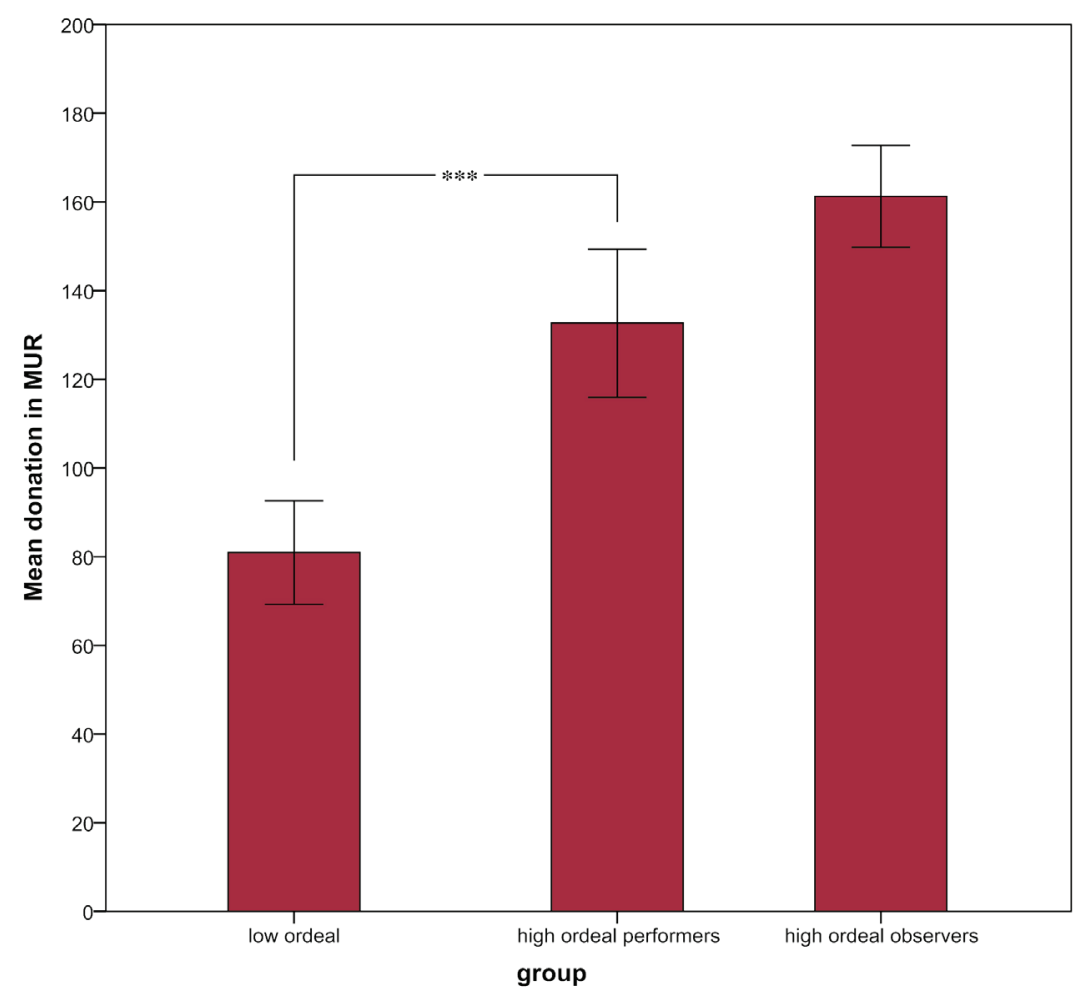

FIGURE 2 Mean donations among the different groups. ${ }^{* * *} p<0.001$. Error bars represent SEM. 
in the high-ordeal group donated an average of 150.59 rupees $(\mathrm{SD}=68.74)$ and those in the low-ordeal group donated 80.91 rupees $(\mathrm{SD}=69.18)$. The difference between the high-ordeal and the low-ordeal group was significant: $t(84)=4.61$, $p<0.001$. Within the high-ordeal group, there were no significant differences between the donations of performers $(132.63$ rupees, $S D=72.79)$ and those of observers (161.25 rupees, $\mathrm{SD}=65.04)$.

More importantly, perceptions of pain were significantly correlated with the amount donated $(r=0.36, p<0.01)$. The more pain individuals perceived in the ritual, the higher their donations to the temple. This provided the first empirical support in a real-life context for previous theoretical speculations that high-ordeal rituals function as social bonding mechanisms. It is the shared experience of pain in a social context that forms a crucial element in this social coordination process of extreme rituals. Extreme rituals can be seen as a social technology to bind in-groups together, which may shed some light regarding to the temporal depth and world-wide spread of such extreme rituals.

\section{Pain and Religiosity}

In the study by Xygalatas et al. (2013), adjusting for religiosity or how often participants visited the temple did not affect the donation and pain findings (Figure 3). Yet, there was a negative relationship between perceived difficulty of carrying the "kavadi" and how religious individuals felt: $r=-0.28$, $p<0.01, N=88$. In other words, the more religious individuals reported greater ease of performing the high-ordeal acts of worship. In a separate study in Thailand, Fischer and Kruekaew (in preparation) examined high-ordeal participants, their supporters and individuals seeking blessings from highordeal participants during the annual Vegetarian Festival (Figure 4). Similar to the Thaipusam, this is one of the most extreme religious rituals worldwide, with roots reaching back about 5000 years to pre-Chinese devotional rituals. Participants reportedly enter in trance (become spiritual mediums) and are pierced with various objects ranging from skewers, metal rods and swords, to household appliances, tree branches, bicycles, cell phones, beach umbrellas or guns. These high-ordeal participants then process about $20 \mathrm{~km}$ through town and bless families and households while being showered by firecrackers and engaging in further devotional acts of self-mortification. When asked after the ritual about their levels of religiosity and experienced pain of the piercing, a negative correlation emerged for the high ordeal participants: $r=-0.40, p<0.05$, $N=41$. More religious individuals reported less pain (Fischer and Kruekaew, data not shown). Therefore, similar to the findings by Xygalatas et al. (2013), the subjective experience of pain and suffering was negatively associated with 


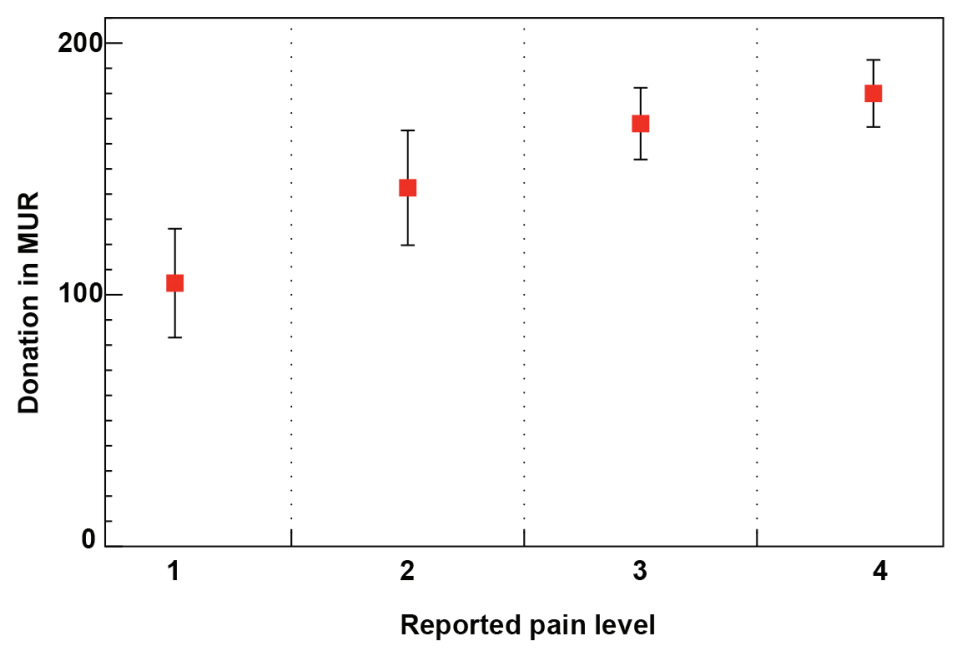

FIGURE 3 Perceived pain divided into quartiles. The linear polynomial contrast on these quartiles is highly significant: $F(1,46)=8.13, p<0.01$, indicating that higher levels of pain are associated with bigger donations. Error bars represent SEM.

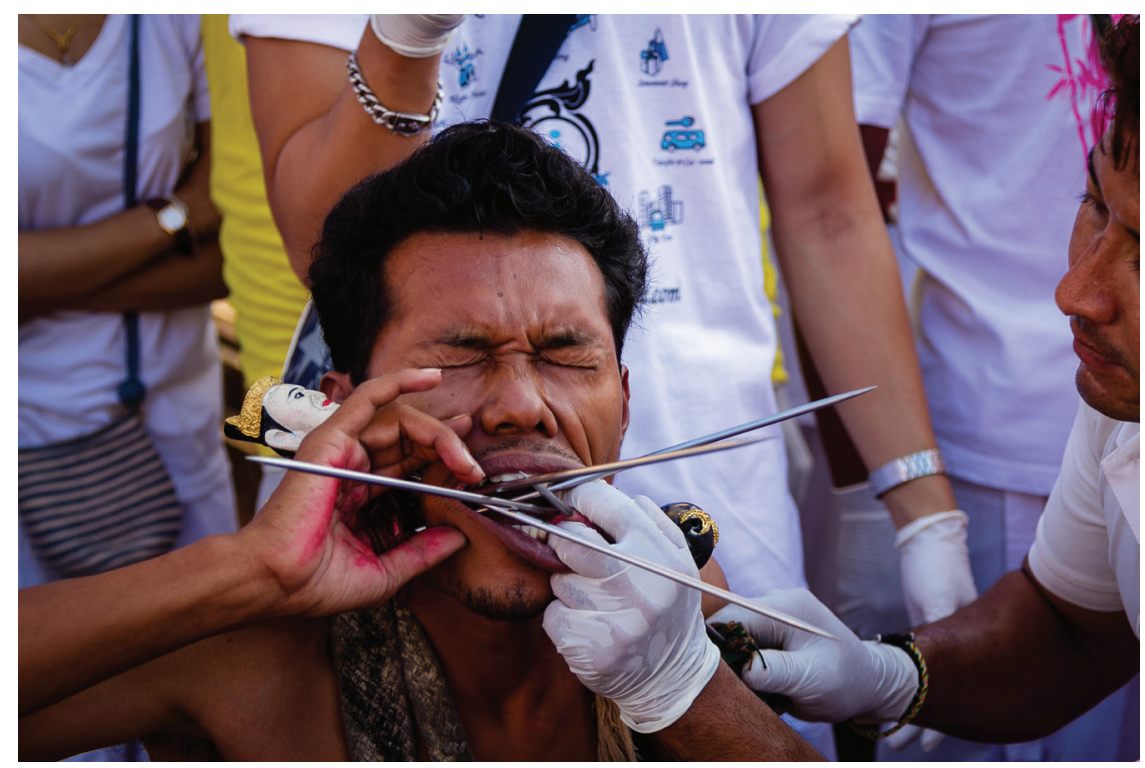

FIGURE 4 Painful rituals: a spirit medium is being pierced during the Vegetarian festival. 
self-reported religiosity. These patterns need to be re-examined in light of the theories and experimental findings of pain experiences reviewed above.

\section{Individual Agency versus Social Functions: Revisiting Group- Selection Arguments}

In the following, we would like to put forward some speculations that connect the experimental and theoretical work on pain with the noted social functions of extreme rituals (Figure 5). Bastian et al. (2011) provided some experimental support for the moral cleansing function of pain. It has often been noted that spiritually impure individuals suffer more during extreme rituals (Cohen, 2001; Xygalatas, 2012). Connecting these lines of experimental research and anthropological observations with the noted negative association between pain, suffering and religiosity in both Hindu and Buddhist contexts presented above, we could speculate that those individuals who feel more guilt or believe that they have committed some moral transgression (they feel they have failed in their religious duties) are more likely to engage in these high-ordeal acts and then experience them as more painful. This experience of pain functions as an opportunity for them to cleanse themselves from the noted transgressions. In addition, this experienced pain, driven by perceptions of moral failure, is then associated with a motivation to donate more to the in-group. Overall, we may expect even greater efforts to conform to and reinforce moral norms (e.g., punish transgressors of perceived norms) among individuals engaging in this high-ordeal rituals due to their motivation to purify and cleanse themselves. For observers, the apparent supernatural powers of the high-ordeal participants may create a sense of awe, leading to greater supernatural beliefs (Valdesolo and Graham, 2014). The increased religiousness should also lead to greater identification with the group of believers and social bonding. In addition, observers with greater levels of empathetic reactions will be more drawn into the group (cf., Konvalinka et al., 2011; Xygalatas et al., 2013) and biologically related observers will be likely to experience strong emotional reactions to the display of ordeal (Konvalinka et al., 2011; Fischer et al., 2014). Therefore, the subjective experience of pain and suffering shared by both observers and participants may be an important psychological mechanism in motivating individuals to perform and witness these rituals as well as the functionality of the rituals at the group level. Therefore, the social context (feelings of personal shortcomings through internalization of social norms and expectations) motivates individual agency of publicly visible high ordeal actions, which then reinforces social bonds in 


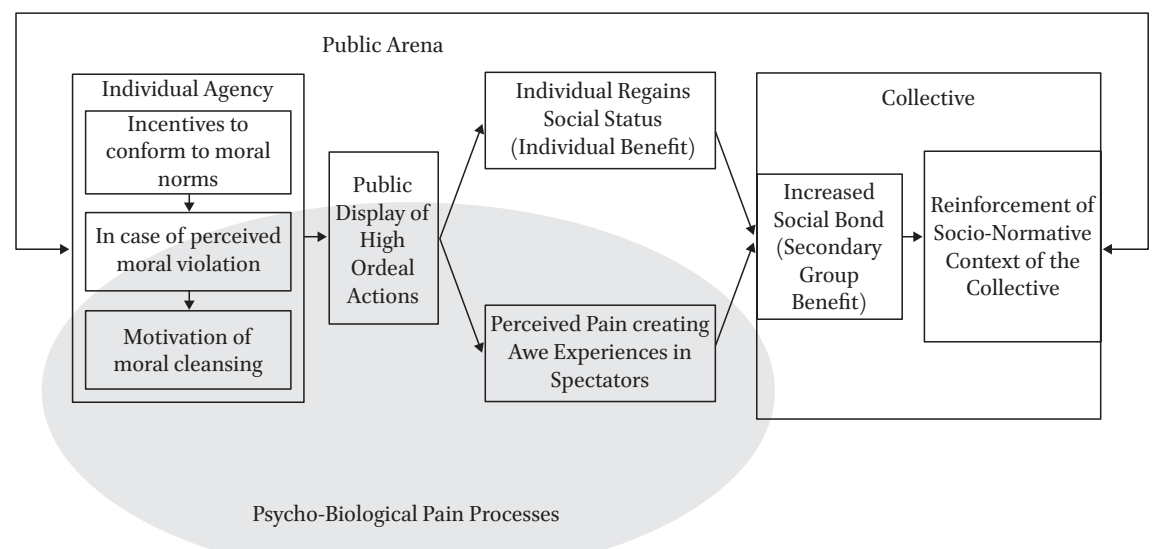

FIGURE 5 Conceptual framework of extreme rituals as social technology: linking individual agency to collectives via public display of high ordeal actions.

the collective and acts as a social reinforcement mechanism. Thus, through the public performance of these devotional acts, individual-level agency that involves complex biological processes of pain perception results into self-reinforcing social mechanisms that bind groups together.

This reasoning leads to interesting implications for group-level selection theories. One of the common arguments is that extreme rituals have survived because groups with rituals that are more effective in bonding the in-group are more likely to survive compared to groups that are lacking such rituals (e.g., Sosis and Bressler, 2003; Henrich, 2009; see also Wilson et al., 2008). According to these theories, there should be selective pressures at the group level that provided some advantage to those groups who had these ritual social technologies compared to those who lacked them. However, our reasoning and preliminary data suggest that a group-level explanation may not be necessary. Given the differential experience of pain, together with the motivational appeal of hurting oneself to achieve moral cleansing, the selective advantage operates primarily at the individual level. Engaging in these rituals allowed people to gain status within the group and avoid marginalization. These acts then have social ramifications by bringing individuals closer together. However, this important group-level outcome would not have been possible without the individuallevel processes of pain perception that are biologically rooted and common to all humans. Our analysis suggests that extreme rituals are tapping on psychobiological pain processes that provide a selective advantage to individuals, which only then provides a secondary advantage at the group level. Put 
differently, the evolutionary benefit is at the individual level with (secondary) consequences at the group level.

In summary, we have argued that extreme rituals are an important social technology that helps bind groups together. Yet, a closer examination of the individual level processes suggests that the selective advantage may lie at the individual level and only secondarily at the group level. After all, these social technologies have to be attractive to the individuals who will perform these acts, otherwise these practices would not have survived through time and across cultures. To test those claims, a multi-level approach is needed. For example, mathematical models and historical analyses may contribute important insights on the long-term transmission and survival of such practices; controlled laboratory studies are needed to examine individual-level mechanisms related to pain and emotion; and carefully designed field studies are necessary to connect group and individual processes as well as to bring contextually relevant findings from real-world rituals. We consider the field experimental research presented here as a first step towards a better understanding of these multi-level processes and their complexities.

\section{References}

Bastian, B., Jetten, J. and Fasoli, F. (2011) Cleansing the soul by hurting the flesh: The guilt-reducing effect of pain. Psychological Science 22: 334-335.

Cohen, E. (2001). The Chinese Vegetarian Festival in Phuket. Religion, ethnicity and tourism on a Southern Thai Island. White Lotus Press, Bangkok.

Dietrich, A. and Audiffren, M. (2011). The reticular-activating hypofrontality (RAH) model of acute exercise. Neuroscience and Biobehavioral Reviews 35, 1305-1325.

Fischer, R., Xygalatas, D., Mitkidis, P., Reddish, P., Tok, P., Konvalinka, I. and Bulbulia, J. (2014). The fire-walker's high: Affect and physiological responses in an extreme collective ritual. PLoS ONE 9, e88355.

Henrich, J. (2009) The evolution of costly displays, cooperation, and religion. Evolution and Human Behavior 30, 244-260.

Konvalinka, I., Xygalatas, D., Bulbulia, J., Schjødt, U., Jegindø, E.-M., Wallot, S., Van Orden, G. and Roepstorff, A. (2011) Synchronized arousal between performers and related spectators in a fire-walking ritual. Proceedings of the National Academy of Sciences of the United States of America 108, 8514-8519.

Melzack, R. (2005). Evolution of the neuromatrix theory of pain. Pain Practice 5, 85-94. Melzack, R. and Wall, P. D. (1965). Pain mechanisms: A new theory. Science 150, 971-979. Sosis, R. and Bressler, E. R. (2003). Cooperation and commune longevity: A test of the costly signaling theory of religion. Cross-Cultural Research 37, 211-239. 
St Clair Gibson, A., Baden, D. A., Lambert, M. I., Lambert, E. V., Harley, Y. X.R., Hampson, D., Russell, V. and Noakes, T. D. (2003). The conscious perception of the sensation of fatigue. Sports Medicine 33: 167-176.

Valdesolo, P. and Graham, J. (2014). Awe, uncertainty and agency detection. Psychological Science 25, 170-178.

Wilson, D. S., Van Vugt, M. and O'Gorman, R. (2008). Multilevel Selection Theory and Major Evolutionary Transitions: Implications for Psychological Science. Current Directions in Psychological Science 17, 6-9.

Xygalatas, D. (2012). The Burning Saints: Cognition and Culture in the Fire-walking Rituals of the Anastenaria. Acumen, London.

Xygalatas, D., Mitkidis, P., Fischer, R., Reddish, P., Skewes, J., Geertz, A. W., Roepstorff, A. and Bulbulia, J. (2013) Extreme rituals promote prosociality. Psychological Science 24, 1602-1605. 